Discrete Comput Geom 38:443-450 (2007)

DOI: $10.1007 / \mathrm{s} 00454-007-1345-4$

\title{
Multiple Coverings of the Plane with Triangles*
}

\author{
Gábor Tardos ${ }^{1,2}$ and Géza Tóth ${ }^{2}$ \\ ${ }^{1}$ School of Computing Science, Simon Fraser University, \\ Burnaby, British Columbia, Canada V5A 1S6 \\ tardos@cs.sfu.ca \\ ${ }^{2}$ Rényi Institute of Mathematics, Hungarian Academy of Sciences, \\ Pf. 127, H-1364 Budapest, Hungary \\ geza@ renyi.hu
}

\begin{abstract}
We prove that any 43 -fold covering of the plane with translates of a triangle can be decomposed into two coverings.
\end{abstract}

\section{Introduction}

Let $\mathcal{C}=\left\{T_{i} \mid i \in I\right\}$ be a collection of planar sets. It is a $k$-fold covering if every point in the plane is contained in at least $k$ members of $\mathcal{C}$. A one-fold covering is simply called a covering.

Definition. A planar set $T$ is said to be cover-decomposable if the following holds. There exists a constant $k=k(T)$ such that every $k$-fold covering of the plane with translates of $T$ can be decomposed into two coverings. Pach [P1] proposed the problem of determining all cover-decomposable sets.

Conjecture (Pach). All convex sets are cover-decomposable.

The conjecture is verified in two special cases.

\footnotetext{
* Gábor Tardos was supported by Hungarian Research Fund Grants OTKA T-046234, AT-048826, and NK-62321 and an NSERC Discovery Grant. Géza Tóth was supported by Hungarian Research Fund Grants OTKA-T-038397 and OTKA-T-046246.
} 


\section{Theorem A.}

(i) [P2] Every centrally symmetric open convex polygon is cover-decomposable.

(ii) [MP] The open unit disc is cover-decomposable.

Based on the ideas of Pach [P2], in this note we prove another special case.

Theorem 1. Every open triangle is cover-decomposable.

In fact we prove the following somewhat stronger statement that, however, requires the technical condition that the collection of translates is locally finite, i.e., that every compact region intersects a finite number of the translates.

Theorem 2. Every locally finite collection $\mathcal{C}$ of translates of the same (open or closed) triangle $T$ can be partitioned into two parts such that every point that is covered by at least 43 of the triangles in $\mathcal{C}$ is covered by a triangle in both parts.

We believe that Theorem 2 remains true without the requirement that $\mathcal{C}$ is locally finite, however our proof uses this assumption. If Theorem 2 is true without this assumption, then, clearly, closed triangles are also cover-decomposible. We find it hard to imagine that closed triangles are not cover-decomposible, but a proof of this seems to be elusive.

We finish this introductory section by observing that Theorem 2 implies Theorem 1. Indeed, from every 43 -fold covering of the plane by translates of the same open triangle (or by arbitrary open sets of bounded diameter) one can select a locally finite subcollection that is also a 43-fold cover. To see this it is enough to use the square grid to cut the the plane into squares and one can use the compactness of the closed squares $S_{i}$ to find a finite subset $\mathcal{C}_{i}$ of $\mathcal{C}$ that is covering $S_{i}$ 43-fold. If we delete all sets from $\mathcal{C}_{i}$ that are disjoint from $S_{i}$, then the union $\bigcup_{i} \mathcal{C}_{i}$ is locally finite and a 43 -fold covering of the plane.

Notice that similar statement for closed triangles is false. It is easy to find a covering of the plane by translates of a closed triangle that does not contain a locally finite subset that also covers the plane. One can also find a two-fold covering consisting of translates of a closed triangle such that no proper subset is a two-fold covering and the cardinality of the collection is the continuum.

For examples of not cover-decomposable sets and other related problems see [PTT].

\section{Proof of Theorem 2}

Just like in [P2], we formulate (and solve) the problem in its dual form. Fix $O$, the center of gravity of the triangle $T$, as our origin in the plane. For a planar set $P$ and a point $x$ in the plane we use $P(x)$ to denote the translate of $P$ by the vector $\overrightarrow{O x}$. Let $\bar{T}$ be the reflection through $O$ of the triangle $T$. Consider any collection $\mathcal{C}=\left\{T\left(x_{i}\right) \mid i \in I\right\}$ of translates of $T$. For any point $p, p \in T\left(x_{i}\right)$ if and only if $x_{i} \in \bar{T}(p)$. To see this, apply a reflection through the midpoint of the segment $p x_{i}$. This switches $T\left(x_{i}\right)$ and $\bar{T}(p)$, and also switches $x_{i}$ and $p$.

The collection $\mathcal{C}$ covers $p$ at least 43 times if and only if $\bar{T}(p)$ contains at least 43 elements of the set $\mathcal{S}=\left\{x_{i} \mid i \in I\right\}$. The required partition of $\mathcal{C}$ exists if and only if the 
set $\mathcal{S}$ can be colored with two colors such that every translate of $\bar{T}$ that contains at least 43 elements of $\mathcal{S}$ contains at least one element of each color.

It is easy to see that $H=T \cap \bar{T}$ is a hexagon whose vertices are those points which divide the sides of $T$ in the ratio $1: 2$. Observe that the plane has a lattice tiling with translates of this hexagon. We can assume without loss of generality that each $x_{i}$ is in the interior of some hexagon. By our assumption on local finiteness each hexagon contains finitely many points $x_{i}$.

Elementary analysis shows that any translate of $\bar{T}$ intersects at most six of the hexagons in the tiling, so if it contains at least 43 elements of $\mathcal{S}$, then it contains at least eight elements belonging to the same hexagon.

Let $A, B$, and $C$ be the vertices of $\bar{T}$. Let us consider the natural linear order on the lines of the plane that are parallel to the side $B C$ with the line through $A$ defined smaller than the line $B C$. We define the partial order $<_{A}$ on the points with $x<_{A} y$ if the line through $x$ is smaller than the line through $y$. We have $A<_{A} B$ and thus also $A<_{A} C$. Similarly consider the partial ordering $<_{B}$ according to the lines parallel to $A C$ with $B<_{B} C$, and the partial ordering $<_{C}$ according to the lines parallel to $A B$ with $C<_{C} A$. We define $W_{A}$ to be the set of points $p$ with $O<_{B} p$ and $O<_{C} p$. Similarly, let $W_{B}$ be the set of points $p$ with $O<_{C} p$ and $O<_{A} C$ and let $W_{C}$ be the set of points $p$ with $O<_{A} p$ and $O<_{B} p$. For $X=A, B$, or $C$ the set $W_{X}$ is an open wedge with $O$ as the apex, and it the smallest such wedge such that $W_{X}(X)$ contains $\bar{T}$.

Since the hexagon $H$ intersects at most two sides of any translate of $\bar{T}$, the intersection of $H$ with any translate of $\bar{T}$ is equal to the intersection of $H$ with a suitable translate of $W_{A}, W_{B}$, or $W_{C}$. This is immediate for open triangles $T$ and $\bar{T}$. For closed triangles one should consider the closures of the cones. However, for any finite set $\mathcal{S}$ and $X=A, B$, or $C$ the intersection of a translate of the closure of $W_{X}$ with $\mathcal{S}$ can be obtained as the intersection of $\mathcal{S}$ with another translate of $W_{X}$, so we can consider the open wedges in this case too.

We finish the proof of Theorem 2 by separately coloring the subsets of $\mathcal{S}$ belonging to each hexagon in the tiling. Each such subset is colored by the coloring guaranteed by Theorem 3. This results in a coloring required by the dual form of Theorem 2 . This finishes the proof of Theorem 2 provided we prove the following Theorem 3.

Theorem 3. Any finite set $\mathcal{S}$ of points in the plane can be colored with two colors such that any translate of $W_{A}, W_{B}$, or $W_{C}$ which contains at least eight of the points, contains at least one of each color.

Proof. Notice first that a small enough perturbation of the points in $\mathcal{S}$ may introduce new intersections with translates of $W_{A}, W_{B}$, and $W_{C}$ but will not result in the loss of such sets. Therefore we may and will assume without loss of generality that the points in $\mathcal{S}$ are in general position, i.e., no two points in $\mathcal{S}$ determine a line parallel to any one of the sides of the triangle $\bar{T}$. In other words, we assume that $<_{A},<_{B}$, and $<_{C}$ are linear orders on $\mathcal{S}$.

For $X=A, B$, or $C$ we call a point $x \in \mathcal{S}$ an $X$-boundary point if $W_{X}(x) \cap \mathcal{S}=\emptyset$. In other words, $x$ is an $X$-boundary point if translating $W_{X}$ such that its apex moves to $x$, the translate is disjoint from $\mathcal{S}$. See Fig. 1(a). We call a point in $\mathcal{S}$ a boundary point of $\mathcal{S}$ if it is an $X$-boundary point for $X=A, B$, or $C$. Note that the same point can be 


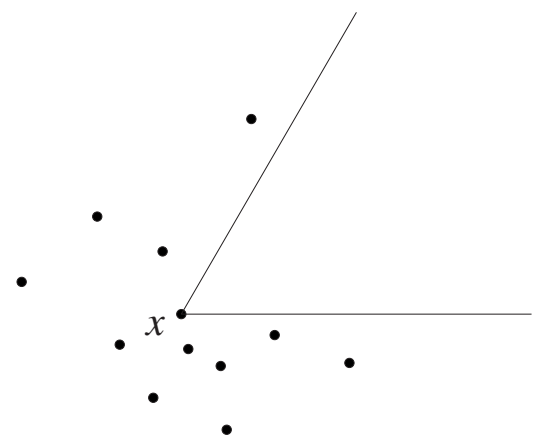

(a)

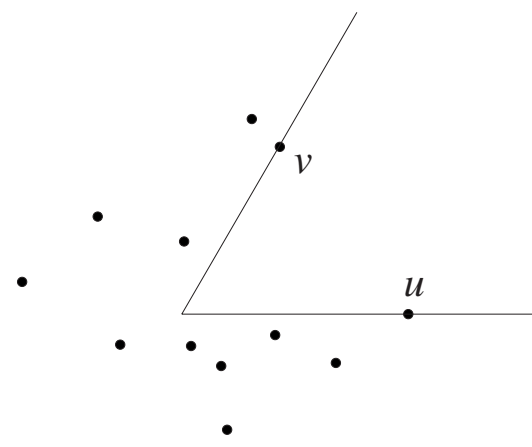

(b)

Fig. 1. (a) $x$ is an $A$-boundary point. (b) $u, v$ are $A$-neighbors.

a boundary point of more than one type, for instance, it can be an $A$-boundary and a $B$-boundary point at the same time.

Notice that $<_{B}$ and $<_{C}$ give the opposite linear order on $A$-boundary points. When speaking of $A$-boundary elements we always consider them with one of these orderings. In particular, we call two points of $\mathcal{S} A$-neighbors if both of them are $A$-boundary points and there is no $A$-boundary point between them according to the ordering $<_{B}$ (or, equivalently, according to $<_{C}$ ). See Fig. 1(b) for a geometric interpretation of this concept. We use $B$-neighbors and $C$-neighbors in an analogous meaning. We call two points of $\mathcal{S}$ neighbors if they are $X$-neighbors for some $X=A, B$, or $C$.

Notice that any translate $W_{A}(p)$ of the wedge $W_{A}$ that contains any element of $\mathcal{S}$ contains at least one $A$-boundary point and the $A$-boundary points in $W_{A}(x)$ form an interval of the set of all $A$-boundary points in the ordering $<_{B}$. Notice also that the smallest elements of $\mathcal{S}$ in the linear orderings $<_{B}$ and $<_{C}$ are $A$-boundary points, these are the two extremal $A$-boundary points.

Similar observations hold for $B$ - and $C$-boundary points of $\mathcal{S}$. As a consequence one may notice that the minimal elements in $\mathcal{S}$ according to the orders $<_{A},<_{B}$ and $<_{C}$ are $X$-boundary elements of at least two different values of $X$ and there is at most a single element of $\mathcal{S}$ that is an $X$-boundary point for all three possible values of $X$.

Let $X=A, B$, or $C$. We call a point $x \in \mathcal{S} X$-rich if $x$ is an $X$-boundary point, there is a translate $W_{X}(p)$ of $W_{X}$ containing $x$ such that $\left|\mathcal{S} \cap W_{X}(p)\right| \geq 8$, and $x$ is the only $X$-boundary point in $W_{X}(p)$. We call a point in $\mathcal{S}$ rich if it is $X$-rich for some $X=A$, $B$, or $C$.

We call a coloring of $\mathcal{S}$ to blue and red acceptable if all rich points are colored blue and between any pair of neighbors at least one is blue.

We say that a point $x \in \mathcal{S}$ is good for a particular acceptable coloring of $\mathcal{S}$ if it is red or it is rich or for $X=A, B$, or $C x$ has a red $X$-neighbor $y$ with $y<_{X} x$. If a point of $\mathcal{S}$ is not good for an acceptable coloring we call it bad.

The following three claims finish the proof of Theorem 3 and therefore also the proof of Theorems 1 and 2. Claims 1 and 2 show that the coloring established in Claim 3 satisfies the requirements of Theorem 3 . 
Claim 1. Consider an acceptable coloring of $\mathcal{S}$. Every wedge of the form $W_{X}(p)$ that contains at least eight points of $S$ contains a blue point of $\mathcal{S}$.

Proof. If $W_{X}(p)$ contains any points of $\mathcal{S}$ it contains a non-empty interval of $X$ boundary points. If this interval consists of more than a single point, then it contains a pair of $X$-neighbors and one of these points is blue. If, however, $W_{X}(p)$ contains a single $X$ boundary point $x$ but contains at least eight points of $\mathcal{S}$, then $x$ is rich by definition and therefore it is blue.

Claim 2. Consider an acceptable coloring of $\mathcal{S}$. If a wedge of the form $W_{X}(p)$ contains at least eight points of $\mathcal{S}$, then it contains at least four bad points or at least one red point.

Proof. Assume without loss of generality that $X=A$. By moving $p$ we can shrink $W_{A}(p)$ so we may assume without loss of generality that $S=W_{A}(p) \cap \mathcal{S}$ has exactly eight points. If $S$ contains a red point we are done, so assume all points in $S$ are blue. Suppose that $x \in S$ is good. We claim that either $x$ is the first or last $A$-boundary point in $S$, or $x$ is the $<_{A}$-minimal $B$-boundary point in $S$, or else $x$ is the $<_{A}$-minimal $C$-boundary point in $S$.

To see this notice first that $x$, being blue and good, must be a boundary point, moreover, for $Y=A, B$, or $C$, either $x$ is $Y$-rich or $x$ has a red $Y$-neighbor $y$ with $y<_{Y} x$. The cases of the following case analysis are illustrated in Fig. 2.

Assume first that $Y=A$. If $x$ is not the first or last $A$-boundary point in $S$, then any translate of $W_{A}$ containing $x$ but not containing other $A$-boundary points must be contained in $W_{A}(p)$ and therefore $x$ is not $A$-rich. Both $A$-neighbors of $x$ are contained in $W_{A}(p)$, so neither is red.
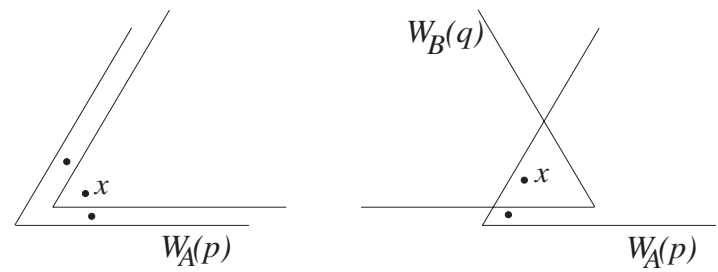

(a)

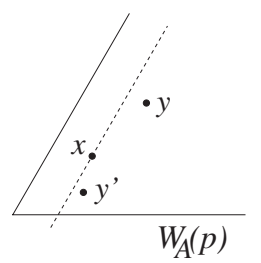

(b)

Fig. 2. (a) $x$ cannot be rich. (b) If $y$ is a $B$-neighbor of $x$ with $y<_{B} x$, then $y \in W_{A}(p)$. 
In the remaining case $Y=B$ or $C$ and we assume by symmetry that that $Y=B$. Now we assume that $x$ is not the $<_{A}$-minimal $B$-boundary point in $W_{A}(p)$. Consider a translate $W_{B}(q)$ of $W_{B}$ containing $x$ but no other $B$-boundary points. It is easy to see that $W_{B}(q) \cap \mathcal{S}$ is contained in $W_{A}(p)$ so $x$ cannot be $B$-rich. If $x$ has a $B$-neighbor $y$ with $y<_{B} x$, then another easy argument shows $y \in W_{A}(p)$ and therefore $y$ must be blue.

Claim 3. There is an acceptable coloring of $\mathcal{S}$ for which there are at most three bad points.

Proof. We give a construction for such a coloring. Naturally, we start by coloring red all points of $\mathcal{S}$ that are not boundary points and by coloring blue all rich points.

Let $z_{A}$ be the $<_{A}$-maximal among the points of $\mathcal{S}$ that are both $B$ - and $C$-boundary points. The $<_{A}$-minimal point in $\mathcal{S}$ has this property, therefore $z_{A}$ exists. Similarly, let $z_{B}$ be the $<_{B}$-maximal point among the points of $\mathcal{S}$ that are simultaneously $A$ and $C$-boundary points and let $z_{C}$ be the $<_{C}$-maximal among the points of $\mathcal{S}$ that are simultaneously $A$ - and $B$-boundary points. Note that these points may coincide. See Fig. 3.

We partition $\mathcal{S} \backslash\left\{x_{A}, x_{B}, x_{C}\right\}$ into four sets as follows. For $X=A, B$, or $C$ we let $\mathcal{S}_{X}=\left\{x \in \mathcal{S} \mid x<_{X} z_{X}\right\}$. We let $\mathcal{S}_{0}=\left(\mathcal{S} \backslash\left\{z_{A}, z_{B}, z_{C}\right\}\right) \backslash\left(\mathcal{S}_{A} \cup \mathcal{S}_{B} \cup \mathcal{S}_{C}\right)=\{x \in \mathcal{S} \mid$ $\left.z_{A}<_{A} x, z_{B}<_{B} x, z_{C}<_{C} x\right\}$. It is not hard to verify that this is indeed a partition. Note that each of these sets may be empty.

We color $z_{A}, z_{B}$, and $z_{C}$ blue, then we color the boundary points of the four parts separately. For $X=A, B$, or $C$ and $Y=A, B, C$, or 0 we take the $X$-boundary points in $\mathcal{S}_{Y}$ and consider them in increasing order according to $<_{X}$. If we get to a point that is not colored we color it red and we color every neighbor of it blue. This goes for every $Z$-neighbor for arbitrary $Z$, for example, a point in $\mathcal{S}_{A}$ can have two $B$-neighbors and
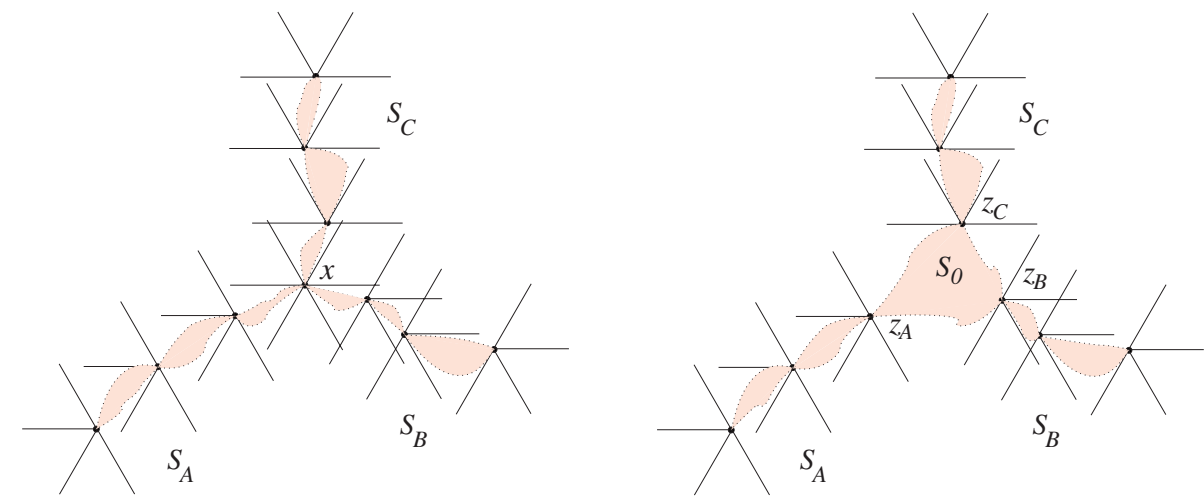

Fig. 3. The boundary vertices and the partition of $\mathcal{S}$. On the left we have $x=z_{A}=z_{B}=z_{C}$. 
two $C$-neighbors. These neighbors may have already been colored blue (because they are rich, because they are one of $z_{A}, z_{B}$, or $z_{C}$, or because of an earlier red neighbor) but they are not colored red as any neighbor of any red point is immediately colored blue. This same observation proves that the resulting coloring is acceptable.

Notice that the coloring processes for the four parts $\mathcal{S}_{A}, \mathcal{S}_{B}, \mathcal{S}_{C}$, and $\mathcal{S}_{0}$ are independent, no neighbor of a point in one part lies in another. The three coloring procedures for $\mathcal{S}_{0}$ are also independent, because boundary points here have only one type. In $\mathcal{S}_{A}$ there are no $A$-boundary points, but there may be several points that are $B$ - and $C$-boundary points simultaneously. Therefore the actual coloring of $\mathcal{S}_{A}$ may depend on which process reaches these common points first. Synchronize the two coloring processes on $\mathcal{S}_{A}$ so that they reach the common points at the same time. This is possible, as their order is the same in the two ordering $<_{B}$ and $<_{C}$. We assume similar synchronizations of the coloring processes of $\mathcal{S}_{B}$ and $\mathcal{S}_{C}$.

We finish the proof by observing that with the possible exception of $z_{A}, z_{B}$, and $z_{C}$ every point of $\mathcal{S}$ is good for the coloring obtained. To see this notice that we only colored a point $x$ other than $z_{A}, z_{B}$, or $z_{C}$ blue if it was rich or if one of its neighbors $y$ were colored red. However, the order in which these points were considered ensures that if $x$ and $y$ are $X$-neighbors then $y<_{X} x$ as needed.

Remarks. The value 43 in Theorem 2 is probably far from being optimal. We cannot even rule out the possibility that the result holds with 3 instead of 43 . An obvious route for improvement is the strengthening of Claim 3 such that it allows for a single bad point only. This would improve the constant 8 in Theorem 3 to 6 and the constant 43 in Theorem 2 to 31 . Note, however, that some sets $\mathcal{S}$ do not allow for an acceptable coloring without any bad vertices.

By hardly any modification of our argument one can prove the following generalization of Theorem 3.

Theorem 3'. For any positive integer $k$, any finite set $\mathcal{S}$ in the plane can be colored red and blue such that any translate of $W_{A}, W_{B}$, or $W_{C}$ which contains at least $5 k+3$ of the points contains a blue point and at least $k$ red points.

Using Theorem $3^{\prime}$, one can re-color the red points recursively to obtain the following generalization of Theorems 1 and 2 for more than two colors.

Theorem 4. For every $m>0$ the following two statements hold. Every locally finite collection $\mathcal{C}$ of translates of the same triangle $T$ can be partitioned into $m$ parts such that for every point that is covered by at least $\frac{21}{2} 5^{m}-\frac{19}{2}$ of the triangles in $\mathcal{C}$ is covered by $a$ triangle in each of the parts. Every $\left(\frac{21}{2} 5^{m}-\frac{19}{2}\right)$-fold covering of the plane by translates of the same open triangle can be partitioned into $m$ coverings.

We believe that the exponential bound in Theorem 4 is far from being optimal. It is possible that the statement is true with a linear bound in $m$. 


\section{References}

[MP] P. Mani, J. Pach, Decomposition problems for multiple coverings with unit balls (manuscript, 1986).

[P1] J. Pach, Decomposition of multiple packing and covering, 2, Kolloquium über Diskrete Geometrie, Salzburg (1980), pp. 169-178.

[P2] J. Pach, Covering the plane with convex polygons, Discrete \& Computational Geometry 1 (1986), $73-81$.

[PTT] J. Pach, G Tardos, G. Tóth, Indecomposable coverings, in: The China-Japan Joint Conference on Discrete Geometry, Combinatorics and Graph Theory (CJCDGCGT 2005), Lecture Notes in Computer Science 4381, Springer, Berlin, 2007, pp. 135-148.

Received April 26, 2006, and in revised form September 19, 2006. Online publication July 13, 2007. 